\title{
Improving entanglement of even entangled coherent states by a coherent superposition of photon subtraction and addition
}

\author{
Shi-You Liu, ${ }^{1}$ Jie-Hui Huang, ${ }^{1} \mathrm{Li}-Y u n \mathrm{Hu}^{1,2}$, Zheng-Lu Duan ${ }^{1}$, Xue-Xiang Xu and Ying-Hua $\mathrm{Ji}^{1,2}$ \\ ${ }^{1}$ Center for Quantum Science and Technology, Jiangxi Normal University, Nanchang 330022, China and \\ ${ }^{2}$ Key Laboratory of Optoelectronic and Telecommunication, Jiangxi Normal University, Nanchang 330022

\begin{abstract}
A new entangled quantum state is introduced by applying local coherent superposition $\left(r a^{\dagger}+t a\right)$
Keywords: quantum entanglement, coherent photon addition and subtraction, IWOP method PACS numbers: 03.65 -a. 42.50.-p
\end{abstract} \\ of photon subtraction and addition to each mode of even entangled coherent state (EECS) and the \\ properties of entanglement are investigated. It is found that the Shchukin-Vogel inseparability, the \\ degree of entanglement and the average fidelity of quantum teleportation of the EECS can be improved \\ due to the coherent superposition operation. The effects of improvement by coherent superposition \\ operation are better than those by single $\left(a^{\dagger}\right)$ and two-photon $\left(a^{\dagger} b^{\dagger}\right)$ addition operations under a small \\ region of amplitude.
}

\section{INTRODUCTION}

Quantum entanglement, as an important resource, plays a critical role in quantum computation and quantum information processing [1]. The two-mode squeezed vacuum state exhibits quantum entanglement between the idle mode and the signal mode, and is often used to as an entangled resource. However, the source of practically available is characteristics of an finite degree of entanglement. In order to realize the long-distance communication schemes of quantum information, entanglement resources with higher degree of entanglement are usually required. Thus it is not trivial to enhance the entanglement of given entangled states.

For this purpose, many theoretical and experimental protocols are proposed to generate such entangled states, which are plausible ways to obtain an entangled state with higher entanglement degree by nonGaussian operations, such as adding or subtracting photons to/from quantum states [2]. For instance, the entanglement between Gaussian entangled states can be increased by subtraction of single photon from Gaussian quadrature-entangled light [3, 4]. The fidelity of quantum teleportation may be enhanced by subtracting one-photon from each mode rather than only one mode [5-7]. In addition, the combination of photon-subtraction and photon-addition operation has been used to generate nonclassical states. For example, nonclassical states of optical field generated by photon subtraction-then-addition or addition-then-subtraction operation [8]. Then multi-photons subtraction-thenaddition or addition-then-subtraction is further operated on coherent states. The nonclassicality of these states are discussed according to photon-number distribution, Mandel Q parameter and Wigner function [9]. On the other hand, it is interesting to note that coherent superposition of non-Gaussian operations is also proposed to manipulate and enhance the entanglement of quantum states [10, 11]. In Ref.[10], coherent superposition of photon subtraction and addition, i.e., the $t a+r a^{\dagger}$ operator is used to transform a classical state to a nonclas- sical one. Then in Ref. [11] the coherent superposition is further performed on two-mode squeezed vacuum for enhancing quantum entanglement or non-Gaussian entanglement distillation. Recently, the entanglement of a non-Gaussian entangled state is investigated by local photon subtraction operation [12]. Thus these coherent superposition operations can be seen as a useful tool in quantum information processing.

In this paper, we will introduce a kind of entangled state, coherent superposition (CS) even entangled coherent state (EECS) $\left|\Psi_{+}(\alpha, 0)\right\rangle$ (see below Eq.(1)), generated by applying coherent superposition operation $\left(t_{A} a+r_{A} a^{\dagger}\right)^{m}\left(t_{B} b+r_{B} b^{\dagger}\right)^{n}$ to each local mode of twomode EECSs and investigate the properties of entanglement. The photon-subtraction $\left(a^{m} b^{n}, r_{A}=r_{B}=0\right)$ EECS and the photon-addition $\left(a^{\dagger m} b^{\dagger n}, r_{A}=r_{B}=1\right)$ EECS can be considered as two special cases of the CSEECSs. Practically speaking, we investigate the degree of entanglement by using the concurrence, the inseparability by using Shchukin-Vogel (SV) criteria, and the average fidelity of quantum teleportation to explore how these properties can be enhanced by the CS operation. It is shown that all these entanglement characteristics can be improved by operating the CS operation on the EECSs under a certain amplitude region. Sa far we know, there is no report about this issue in the literature to date.

This paper is arranged as follows. In section 2 , we shall introduce the CS-EECSs, and derive the normalization factor by using the generating function of single-variable Hermite polynomials, which is necessary for further discussing the entanglement properties of quantum states. It is shown that the factor is just related to Hermite polynomials. The improvement of entanglement measured by Shchukin-Vogel criteria and the concurrence in sections 3 and 4, respectively. Section 5 is devoted to studying the average fidelity of quantum teleportation with continuous variable by using the CS-EECSs as the entangled channel. It is found that the effective teleportation (fidelity $>1 / 2$ ) can be enhanced by CS operation on the EECSs in a small amplitude region. The main results are involved in the last section. 


\section{TWO-MODE COHERENT SUPERPOSITION EECSS}

In this section, we introduce the two-mode CS-EECSs. First, let us begin with the EECSs [13], which is defined by

$$
\left|\Psi_{+}(\alpha, 0)\right\rangle=N_{+, \alpha}(|\alpha, \alpha\rangle+|-\alpha,-\alpha\rangle),
$$

where $|\alpha, \alpha\rangle \equiv|\alpha\rangle_{a} \otimes|\alpha\rangle_{b}$ with $|\alpha\rangle_{a}$ and $|\alpha\rangle_{b}$ being the usual coherent states in $a$ and $b$ modes, respectively, and $\left(N_{+}(\alpha, 0)\right)^{-2}=2\left[1+e^{-4|\alpha|^{2}}\right]$ is the normalization constants. By performing repeatedly the two-mode CS operations $\mathfrak{A}_{m} \equiv\left(r_{A} a^{\dagger}+t_{A} a\right)^{m}$ and $\mathfrak{B}_{n} \equiv\left(r_{B} b^{\dagger}+t_{B} b\right)^{n}$ on the ECSs, one can obtain the output state as

$$
\left|\Psi_{+}(\alpha, m, n)\right\rangle=N_{+, m, n} \mathfrak{A}_{m} \mathfrak{B}_{n}(|\alpha, \alpha\rangle+|-\alpha,-\alpha\rangle),
$$

where $N_{+, m, n}$ represents the normalization factor and $\left|t_{j}\right|^{2}+\left|r_{j}\right|^{2}=1(j=A, B)$. In particular, when $t_{A}=t_{B}=0$ and $r_{A}=r_{B}=1$, Eq.(2) just reduces to the two-mode excited EECSs [14, 15], $\left|\Psi_{+}(\alpha, m, n)\right\rangle=$ $N_{+, m, n} a^{\dagger m} b^{\dagger n}(|\alpha, \alpha\rangle+|-\alpha,-\alpha\rangle)$. The properties of entanglement of single-mode excited EECSs (say $n=0$ ) is discussed in details [16].

Next, we derive the normalization factor $N_{+, m, n}$, which is important for further discussing the properties of statistics and entanglement for quantum states. For this purpose, using the formula $e^{A+B}=e^{A} e^{B} e^{-[A, B] / 2}=$ $e^{B} e^{A} e^{[A, B] / 2}$, which is valid for $[A,[A, B]]=[B,[A, B]]=$ 0 , one can find, for instance,

$$
\begin{aligned}
& \left(r_{A} a^{\dagger}+t_{A} a\right)^{m}|\alpha\rangle \\
& =\left.e^{-\frac{1}{2}|\alpha|^{2}} \frac{\partial^{m}}{\partial s^{m}} e^{\frac{1}{2} s^{2} t_{A} r_{A}+\alpha s t_{A}} e^{\left(s r_{A}+\alpha\right) a^{\dagger}}|0\rangle\right|_{s=0},
\end{aligned}
$$

which leads to (for simplicity, $t_{i}$ and $r_{i}$ are considered as real)

$$
\begin{aligned}
A_{1} & \equiv\left\langle\alpha\left|\left(r_{A} a+t_{A} a^{\dagger}\right)^{m}\left(r_{A} a^{\dagger}+t_{A} a\right)^{m}\right| \alpha\right\rangle \\
& =e^{-|\alpha|^{2}} \frac{\partial^{2 m}}{\partial s^{m} \partial \tau^{m}} e^{\frac{1}{2}\left(s^{2}+\tau^{2}\right) t_{A} r_{A}+\alpha s t_{A}+\alpha^{*} \tau t_{A}} \\
& \times\left.\left\langle 0\left|e^{\left(\tau r_{A}+\alpha^{*}\right) a} e^{\left(s r_{A}+\alpha\right) a^{\dagger}}\right| 0\right\rangle\right|_{s, \tau=0} \\
& =\frac{\partial^{2 m}}{\partial s^{m} \partial \tau^{m}} \exp \left\{\frac{t_{A} r_{A}}{2}\left(s^{2}+\tau^{2}\right)\right\} \\
& \times\left.\exp \left(\tau s r_{A}^{2}+s\left(t_{A} \alpha+r_{A} \alpha^{*}\right)+\tau\left(t_{A} \alpha^{*}+r_{A} \alpha\right)\right)\right|_{s, \tau=0}
\end{aligned}
$$

Using the following formula [17]

$$
\begin{aligned}
& \frac{\partial^{2 m}}{\partial t^{m} \partial s^{m}} \exp \left[R_{1} s+R_{1}^{*} t+R_{3} t s+R_{4}\left(t^{2}+s^{2}\right)\right]_{t=s=0} \\
& =\sum_{l=0}^{m} \frac{R_{3}^{l} R_{4}^{m-l}(m !)^{2}}{l ![(m-l) !]^{2}}\left|H_{m-l}\left[R_{1} /\left(2 i \sqrt{R_{4}}\right)\right]\right|^{2}
\end{aligned}
$$

where $H_{m}(x)$ is the single-variable Hermite polynomials, one can put Eq. (4) into the following form

$$
A_{1}=\sum_{l=0}^{m} \frac{(m !)^{2} t_{A}^{m-l} r_{A}^{m+l}}{2^{m-l} l ![(m-l) !]^{2}}\left|H_{m-l}\left[\bar{\alpha}_{A}\right]\right|^{2} .
$$

Here we have set $\bar{\alpha}_{A}=\left(t_{A} \alpha+r_{A} \alpha^{*}\right) /\left(i \sqrt{2 t_{A} r_{A}}\right)$. In a similar way, one can derive

$$
\begin{aligned}
A_{2} & \equiv\left\langle-\alpha\left|\left(r_{A} a+t_{A} a^{\dagger}\right)^{m}\left(r_{A} a^{\dagger}+t_{A} a\right)^{m}\right| \alpha\right\rangle \\
& =e^{-2|\alpha|^{2}} \sum_{l=0}^{m} \frac{(-1)^{m-l}(m !)^{2} t_{A}^{m-l} r_{A}^{m+l}}{2^{m-l} l ![(m-l) !]^{2}}\left|H_{m-l}\left[\bar{\beta}_{A}\right]\right|^{2},
\end{aligned}
$$

and

$$
\begin{aligned}
B_{1} & \equiv\left\langle\alpha\left|\left(r_{B} b+t_{B} b^{\dagger}\right)^{n}\left(r_{B} b^{\dagger}+t_{B} b\right)^{n}\right| \alpha\right\rangle \\
& =\sum_{l=0}^{n} \frac{(n !)^{2} t_{B}^{n-l} r_{B}^{n+l}}{2^{n-l} l ![(n-l) !]^{2}}\left|H_{n-l}\left[\bar{\alpha}_{B}\right]\right|^{2},
\end{aligned}
$$

as well as

$$
\begin{aligned}
B_{2} & \equiv\left\langle-\alpha\left|\left(r_{B} b+t_{B} b^{\dagger}\right)^{n}\left(r_{B} b^{\dagger}+t_{B} b\right)^{n}\right| \alpha\right\rangle \\
& =e^{-2|\alpha|^{2}} \sum_{l=0}^{n} \frac{(-1)^{n-l}(n !)^{2} t_{B}^{n-l} r_{B}^{n+l}}{2^{n-l} l ![(n-l) !]^{2}}\left|H_{n-l}\left[\bar{\beta}_{B}\right]\right|^{2},
\end{aligned}
$$

where $\quad \bar{\beta}_{A}=\left(r_{A} \alpha-\alpha^{*} t_{A}\right) /\left(i \sqrt{2 t_{A} r_{A}}\right)$, $\bar{\alpha}_{B}=\left(t_{B} \alpha+r_{B} \alpha^{*}\right) /\left(i \sqrt{2 t_{B} r_{B}}\right)$ and $\bar{\beta}_{B}=$ $\left(r_{B} \alpha-\alpha^{*} t_{B}\right) /\left(i \sqrt{2 t_{B} r_{B}}\right)$.

Thus the normalization factor $N_{+, m, n}$ can be given by

$$
\left(N_{+, m, n}\right)^{-2}=2\left(A_{1} B_{1}+A_{2} B_{2}\right) .
$$

It is noted that when $m=n=0$, then $A_{1}=B_{1}=1$, and $A_{2}=B_{2}=e^{-2|\alpha|^{2}}$, thus Eq.(2) becomes the usual EECSs in Eq. (1) with $N_{+, 0,0}^{-2}=2\left[1+\exp \left(-4|\alpha|^{2}\right)\right]$. For the case of $t_{A}=t_{B}=0$ and $r_{A}=r_{B}=1$,corresponding to the two-mode excited EECSs, from Eqs.(4)-(9), one can see that

$$
A_{1}=m ! L_{m}\left(-|\alpha|^{2}\right), B_{1}=n ! L_{n}\left(-|\alpha|^{2}\right),
$$

and

$$
\begin{aligned}
& A_{2}=m ! \exp \left(-2|\alpha|^{2}\right) L_{m}\left(|\alpha|^{2}\right), \\
& B_{2}=n ! \exp \left(-2|\alpha|^{2}\right) L_{n}\left(|\alpha|^{2}\right),
\end{aligned}
$$

thus the normalization factor Eq. (10) reduces to

$$
\begin{aligned}
\left(N_{+, m, n}\right)^{-2} & =2 m ! n !\left[L_{m}\left(-|\alpha|^{2}\right) L_{n}\left(-|\alpha|^{2}\right)\right. \\
& \left.+e^{-4|\alpha|^{2}} L_{m}\left(|\alpha|^{2}\right) L_{n}\left(|\alpha|^{2}\right)\right],
\end{aligned}
$$

where $L_{m}(x)$ is the m-order Laguerre polynomial. Eq. (13) is just the normalization factor of the two-mode excited EECSs.

\section{SHCHUKIN-VOGEL INSEPARABILITY}

For a bipartite continuous-variable state, here we will take the Shchukin-Vogel (SV) criteria [18] to describe the 
inseparability of the CS-EECSs. According to the SV criteria, the sufficient condition of inseparability (entanglement) is given by [19]

$$
S_{+} \equiv\left\langle a^{\dagger} a-\frac{1}{2}\right\rangle\left\langle b^{\dagger} b-\frac{1}{2}\right\rangle-\left\langle a^{\dagger} b^{\dagger}\right\rangle\langle a b\rangle<0 .
$$

Thus we only need to calculate three average values $\left\langle a^{\dagger} a\right\rangle,\left\langle b^{\dagger} b\right\rangle,\langle a b\rangle$ to obtain $S_{+}$.

Here, we consider the property of entanglement of the CS-ECSs with $m=n=1$, $\left|\Psi_{+}(\alpha, 1,1)\right\rangle=N_{+, 1,1} \mathfrak{A}_{1} \mathfrak{B}_{1}(|\alpha, \alpha\rangle+|-\alpha,-\alpha\rangle)$, where $N_{+, 1,1}^{-2}=2\left(A_{1} B_{1}+A_{2} B_{2}\right)$ and

$$
\begin{aligned}
& A_{1}=\left|t_{A} \alpha+r_{A} \alpha^{*}\right|^{2}+r_{A}^{2}, \\
& B_{1}=\left|t_{B} \alpha+r_{B} \alpha^{*}\right|^{2}+r_{B}^{2} \\
& A_{2}=\left(r_{A}^{2}-\left|r_{A} \alpha-\alpha^{*} t_{A}\right|^{2}\right) e^{-2|\alpha|^{2}}, \\
& B_{2}=\left(r_{B}^{2}-\left|r_{B} \alpha-\alpha^{*} t_{B}\right|^{2}\right) e^{-2|\alpha|^{2}} .
\end{aligned}
$$

Under the state $\left|\Psi_{+}(\alpha, 1,1)\right\rangle$, we can derive three average values as

$$
\begin{aligned}
\left\langle a^{\dagger} a\right\rangle_{1,1} & =\frac{O_{A 1} B_{1}+O_{A 2} B_{2}}{A_{1} B_{1}+A_{2} B_{2}}, \\
\left\langle b^{\dagger} b\right\rangle_{1,1} & =\frac{O_{B 1} A_{1}+O_{B 2} A_{2}}{A_{1} B_{1}+A_{2} B_{2}}, \\
\langle a b\rangle_{1,1} & =\frac{R_{A 1} R_{B 1}+R_{A 2} R_{B 2}}{A_{1} B_{1}+A_{2} B_{2}},
\end{aligned}
$$

where $A_{1,2}$ and $B_{1,2}$ are defined in Eqs.(15) and we have set

$$
\begin{aligned}
O_{j 1} & =|\alpha|^{4}+r_{j}^{2}\left(1+3|\alpha|^{2}\right) \\
& +t_{j} r_{j}\left(\alpha^{* 2}+\alpha^{2}\right)\left(1+|\alpha|^{2}\right), \\
O_{j 2} & =e^{-2|\alpha|^{2}}\left[|\alpha|^{4}+r_{j}^{2}\left(1-3|\alpha|^{2}\right)\right. \\
& \left.+t_{j} r_{j}\left(\alpha^{* 2}+\alpha^{2}\right)\left(1-|\alpha|^{2}\right)\right],
\end{aligned}
$$

and

$$
\begin{aligned}
& R_{j 1}=|\alpha|^{2} \alpha+t_{j} r_{j}\left(\alpha^{3}+|\alpha|^{2} \alpha^{*}+\alpha^{*}\right)+2 r_{j}^{2} \alpha, \\
& R_{j 2}=\left[-|\alpha|^{2} \alpha+t_{j} r_{j}\left(\alpha^{3}+|\alpha|^{2} \alpha^{*}-\alpha^{*}\right)+2 r_{j}^{2} \alpha\right] e^{-2|\alpha|^{2}}
\end{aligned}
$$

$$
(j=A, B) .
$$

Using Eqs.(15)-(18) we can get the expression of $S_{+}$. Here we appeal to the numerical calculation for further discussion. In Fig. 2, we plot the sufficient condition of inseparability as the function of $\alpha$ (as a real number) for the EECSs and the CS-EECSs with several different values of $t$. It is shown that the condition $S_{+}<0$ can be satisfied only when $\alpha$ exceeds a certain threshold value

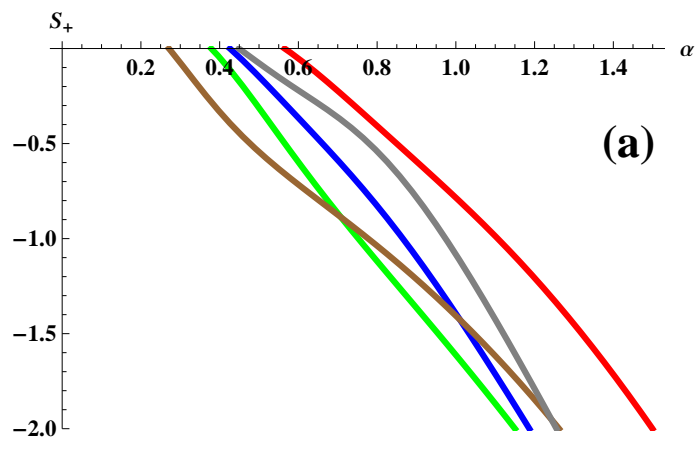

FIG. 1: (Color online) The sufficient condition of inseparability as the function of $\alpha$ for the ECSs (red line) and CS-ECSs with several different values of $t: \mathrm{t}=0$ (Gray line), $\mathrm{t}=0.2$ (Blue line), $\mathrm{t}=0.6$ (Green line) and $\mathrm{t}=0.9$ (Brown).

( $\alpha>0.567$ about). In fact, under the EECSs we have $\left\langle a^{\dagger} a\right\rangle=\left\langle b^{\dagger} b\right\rangle=|\alpha|^{2} \tanh \left(2|\alpha|^{2}\right)$, and $\langle a b\rangle=\alpha^{2}$, thus

$$
S_{+}=\left(|\alpha|^{2} \tanh \left(2|\alpha|^{2}\right)-\frac{1}{2}\right)^{2}-|\alpha|^{4}<0,
$$

which lead to the following condition:

$$
2|\alpha|^{2}\left(\tanh \left(2|\alpha|^{2}\right)+1\right)>1
$$

For the coherent superposition operation $(0<t<1)$, to satisfy the SV criteria, the value of $\alpha$ must be larger than a certain threshold value which decreases with the increasing $t$ (For simplicity we take $t_{A, B}=t$ and $r_{A, B}=r$, $\alpha$ as real). From Fig.1, we can see that (i) these threshold values in CS-EECSs are smaller than that in EECSs; (ii) the inseparability in the CS-EECSs is stronger than in the EECSs and the photon-added EECSs $\left(a^{\dagger} b^{\dagger}\left|\Psi_{+}(\alpha, 0)\right\rangle\right.$, at $t=0$ ); This indicates that the coherent operation on both local modes improves the inseparability better than photon-addition operation $a^{\dagger} b^{\dagger}$ in the small amplitude region. (iii) the photon-subtraction operation $a b$ (at $t=1$ ) can not be used to enhance the inseparability of the EECSs $\left|\Psi_{+}(\alpha, 0)\right\rangle$ due to the fact that $\left|\Psi_{+}(\alpha, 0)\right\rangle$ is the eigenstate of pair annihilation operator $a b$. From the point of SV criteria, the inseparability of the EECSs can be improved better by operating CS on both local modes than photon-addition operation $\left(a^{\dagger} b^{\dagger}\right)$ under a certain condition.

\section{DEGREE OF ENTANGLEMENT OF THE CS-EECSS}

Next, we calculate the amount of entanglement of the CS-EECSs and investigate the influence of the two-mode coherent superposition operation on the entanglement of the CS-EECSs. For continuous-variables-type entangled states like (2), the degree of quantum entanglement of the bipartite entangled states can be measured in terms of the concurrence. Following the approach [20-22], for 
two systems involving nonorthogonal states defined as

$$
|\Psi\rangle=N\left[\mu|\eta\rangle_{a} \otimes|\gamma\rangle_{b}+\nu|\xi\rangle_{a} \otimes|\delta\rangle_{b}\right],
$$

where $|\eta\rangle_{a},|\xi\rangle_{a}$ and $|\gamma\rangle_{b},|\delta\rangle_{b}$ are normalized states of system $a$ and $b$, respectively, the concurrence can be calculated as [23, 24]

$$
C=\frac{2|\mu||\nu|\left[\left(1-\left|p_{1}\right|^{2}\right)\left(1-\left|p_{2}\right|^{2}\right)\right]^{1 / 2}}{|\mu|^{2}+|\nu|^{2}+2 \operatorname{Re}\left(\mu^{*} \nu p_{1} p_{2}^{*}\right)},
$$

where the normalization constant is $N^{-2}=|\mu|^{2}+|\nu|^{2}+$ $2 \operatorname{Re}\left(\mu^{*} \nu p_{1} p_{2}^{*}\right)$, with complex numbers $\mu$ and $\nu$, and $p_{1}=$ ${ }_{a}\langle\eta \mid \xi\rangle_{a}, p_{2}={ }_{b}\langle\delta \mid \gamma\rangle_{b}$. When $\mu=\nu$, the concurrence $C$ becomes

$$
C_{+}=\frac{\sqrt{\left(1-\left|p_{1}\right|^{2}\right)\left(1-\left|p_{2}\right|^{2}\right)}}{1+\operatorname{Re}\left(p_{1} p_{2}^{*}\right)},
$$

which only dependent on the overlaps $\langle\eta \mid \xi\rangle$ and $\langle\gamma \mid \delta\rangle$.

In order to obtain the amount of entanglement of the CS-ECSs, we introduce the following normalized states:

$$
\begin{aligned}
| \pm \alpha\rangle_{m} & =A_{1}^{-1 / 2}\left(r_{A} a^{\dagger}+t_{A} a\right)^{m}| \pm \alpha\rangle, \\
| \pm \alpha\rangle_{n} & =B_{1}^{-1 / 2}\left(r_{B} b^{\dagger}+t_{B} b\right)^{n}| \pm \alpha\rangle,
\end{aligned}
$$

then the CS-ECSs $\left|\Psi_{+}(\alpha, m, n)\right\rangle$ in terms of four normalized states can be reformed as

$$
\begin{aligned}
\left|\Psi_{+}(\alpha, m, n)\right\rangle & =N_{+, m, n}\left(A_{1} B_{1}\right)^{1 / 2} \\
& \left(|\alpha\rangle_{m} \otimes|\alpha\rangle_{n}+|-\alpha\rangle_{m} \otimes|-\alpha\rangle_{n}\right) .
\end{aligned}
$$

Thus one can find

$$
\begin{aligned}
& p_{1}={ }_{m}\langle\alpha \mid-\alpha\rangle_{m}=\frac{A_{2}}{A_{1}}, \\
& p_{2}={ }_{n}\langle-\alpha \mid \alpha\rangle_{n}=\frac{B_{2}}{B_{1}} .
\end{aligned}
$$

Substituting Eq.(26) into Eq.(23) one can get

$$
C_{+}=\frac{\sqrt{\left(A_{1}^{2}-A_{2}^{2}\right)\left(B_{1}^{2}-B_{2}^{2}\right)}}{A_{1} B_{1}+A_{2} B_{2}},
$$

where $A_{1}, B_{1}$ and $A_{2}, B_{2}$ are defined in Eqs.(6)-(9). Eq. (27) is the analytical expression of concurrence for the CS-EECSs.

In particular, for the two-mode excited EECSs, i.e., $t_{A}=t_{B}=0$ and $r_{A}=r_{B}=1$, leading to $A_{1}=$ $L_{m}\left(-|\alpha|^{2}\right), B_{1}=L_{n}\left(-|\alpha|^{2}\right)$ and $A_{2}=e^{-2|\alpha|^{2}} L_{m}\left(|\alpha|^{2}\right)$, $B_{2}=e^{-2|\alpha|^{2}} L_{n}\left(|\alpha|^{2}\right)$, then Eq. (27) reduces to

$$
\begin{aligned}
C_{+} & =\frac{\sqrt{L_{m}^{2}\left(-|\alpha|^{2}\right)-e^{-4|\alpha|^{2}} L_{m}^{2}\left(|\alpha|^{2}\right)}}{L_{m}\left(-|\alpha|^{2}\right) L_{n}\left(-|\alpha|^{2}\right)+e^{-4|\alpha|^{2}} L_{m}\left(|\alpha|^{2}\right) L_{n}\left(|\alpha|^{2}\right)} \\
& \times \sqrt{L_{n}^{2}\left(-|\alpha|^{2}\right)-e^{-4|\alpha|^{2}} L_{n}^{2}\left(|\alpha|^{2}\right)},
\end{aligned}
$$

which exhibits the exchanging symmetry with respect to $m$ and $n$, namely, $C_{+}(\alpha, n, m)=C_{+}(\alpha, m, n)$. In addition, when $m=n$, Eq. (28) becomes

$$
C_{+}=\frac{L_{m}^{2}\left(-|\alpha|^{2}\right)-e^{-4|\alpha|^{2}} L_{m}^{2}\left(|\alpha|^{2}\right)}{L_{m}^{2}\left(-|\alpha|^{2}\right)+e^{-4|\alpha|^{2}} L_{m}^{2}\left(|\alpha|^{2}\right)},
$$

which implies that the two-mode symmetrically excited EECSs is also not a maximally entangled state. As expected, the concurrence of single-mode excited EECSs is just a special case of Eq.(28) [16]. For the two-mode subtraction $\left(t_{A}=t_{B}=1\right.$ and $\left.r_{A}=r_{B}=0\right)$, the concurrence $C_{+}$keep unchanged for the total even number photonsubtraction case ( $m+n=$ even number); while for the total odd number photon-subtraction case $(m+n=$ odd number), the states shall present a transformation from the partially entangled state to the maximum entangled state.

In order to see clearly the effect of different parameters $r_{j}, t_{j}$ and $(m, n)$ on the concurrence of CS-EECSs, $C_{+}$for the state $\left|\Psi_{+}(\alpha, m, n)\right\rangle$ as a function of different parameters are shown in Figs.2-3. We show the concurrence $C_{+}$with a symmetric case of $r_{A}=r_{B}=r$ and $m=n=1,2$ in Fig.2, from which we can see that the concurrence $C_{+}$increases with $r(\alpha)$ when $\alpha(r)$ exceeds a threshold; while the case is still true for $m=n=2$ but without the threshold. Here, for convenience, we plotted only with $\alpha$ being real number.

From Fig.3(a)-(b) with a given value of $r=1 / \sqrt{2}$, one can see that $C_{+}$increases with the increase of $\alpha$ for given parameters $m$ and $n$. Especially, the concurrence $C_{+}$tends to unit for the larger $\alpha$. In addition, for a symmetric case of $m=n, C_{+}$increases with the increase of $m$ (Fig.3(a)); while for an asymmetric case of $m \neq n, C_{+}$increases with the increase of $n$ (Fig.3(b)). Thus the (high-order) coherent superposition operation can be applied to enhance the entanglement of the state $\left|\Psi_{+}(\alpha, m, n)\right\rangle$.

In particular, we make a comparison of the entanglement properties between the CS-EECSs (singlemode CS-EECSs $\left|\Psi_{+}(\alpha, 1,0)\right\rangle$ and $\left.\left|\Psi_{+}(\alpha, 1,1)\right\rangle\right)$ and the single(two-)-mode photon excited EECSs $\left(a^{\dagger}\left|\Psi_{+}(\alpha, 0,0)\right\rangle\right.$ and $a^{\dagger} b^{\dagger}\left|\Psi_{+}(\alpha, 0,0)\right\rangle$ ) (see Fig.4). From Fig.4 one can clearly see that: (i) both photonexcitation and CS operations can improve the entanglement of $\left|\Psi_{+}(\alpha, 0,0)\right\rangle$; (ii) for single-mode operations $\left(t a+r a^{\dagger}\right.$ and $\left.a^{\dagger}\right)$, the effects of the CS operations are more prominent than those of the mere photon-addition in a small-amplitude regime. The threshold value is about $\alpha \lesssim 0.6$ with a symmetrical case of $r=1 / \sqrt{2}$. In addition, the improvement of entanglement is more obvious by an asymmetrical parameter (say $r=0.4$ ) than that by $r=1 / \sqrt{2}$. These results show that applying a CS operator on the EECSs may be better to enhance the quantum entanglement than using only a creation operator on the EECSs, especially for an asymmetrical parameter $r$. For two-mode operations $\left(\left(t a+r a^{\dagger}\right)\left(t b+r b^{\dagger}\right)\right.$ and $\left.a^{\dagger} b^{\dagger}\right)$, the case is true (However, we should point that the most optimal case is not 

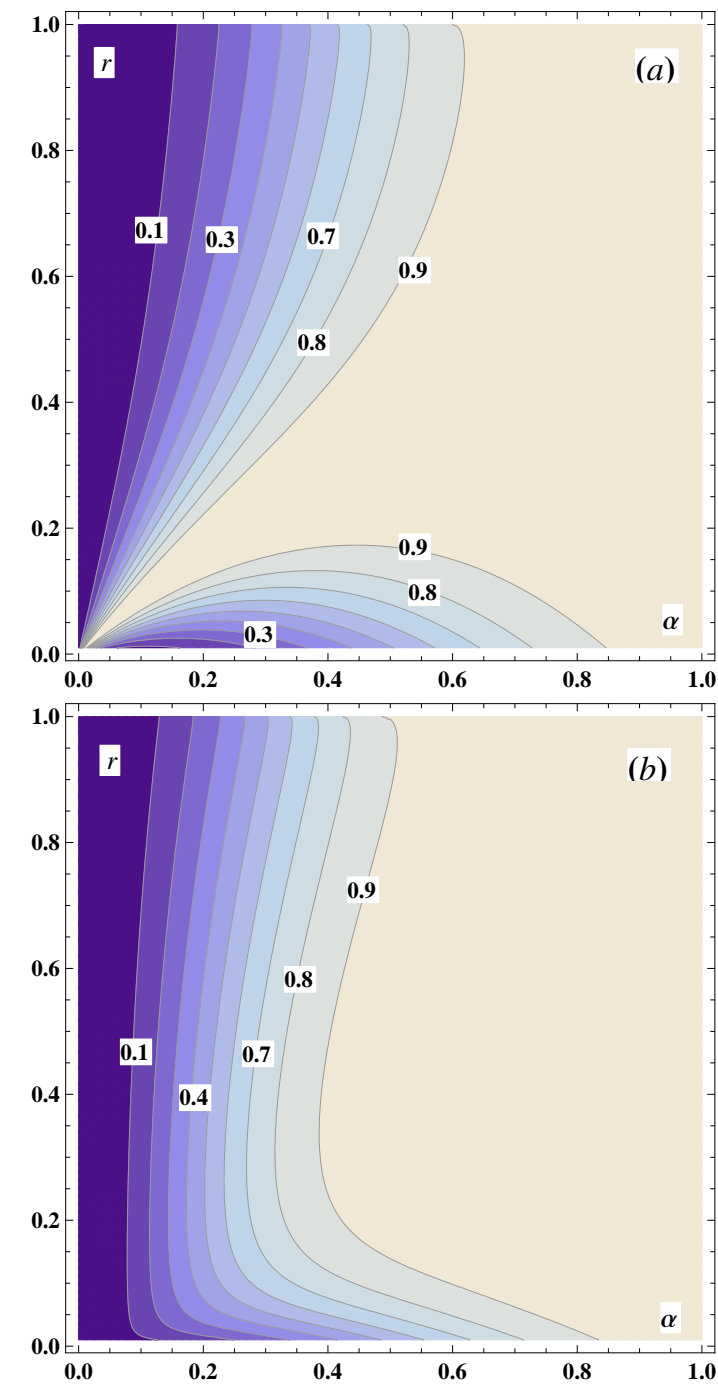

FIG. 2: (Color online) Concurrence of entanglement of $\left|\Psi_{+}(\alpha, m, n)\right\rangle$ as a function of $\alpha$ (considered as a real number) and $r$ for different $m$ and $n$ values. (a) $m=n=1$; (b) $m=n=2$.

for $r=0$ or $t=1$ because two-mode photon-subtraction EECS $a b\left|\Psi_{+}(\alpha, 0,0)\right\rangle$ is $\left|\Psi_{+}(\alpha, 0,0)\right\rangle$ ). (iii) Moreover, the two-mode operations are more effective on increasing the entanglement of EECSs than the single-mode operations. The entanglement can be modulated by different values of $r$, which can also be clearly seen from Fig.3(a).

\section{FIDELITY OF QUANTUM TELEPORTATION}

It is well known that photon subtraction from or photon addition to bipartite Gaussian states can be used to improve the entanglement and quantum teleportation [3, 4, 11, 25-32]. In this section, we consider the effect of coherent superposition on quantum teleportation (QT) by using the CS-EECSs as entangled resource. In
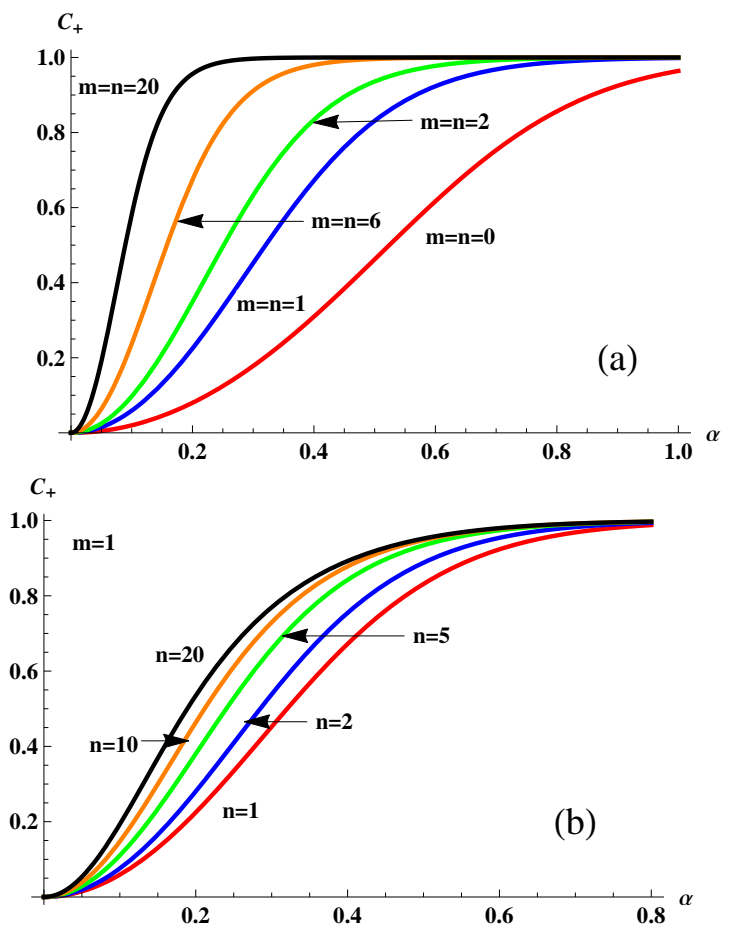

FIG. 3: (Color online) Concurrence of entanglement for the CSEECSs $\left|\Psi_{+}(\alpha, m, n)\right\rangle$ with $r=1 / \sqrt{2}$. (a) $m=n$; (b) $m \neq n$.

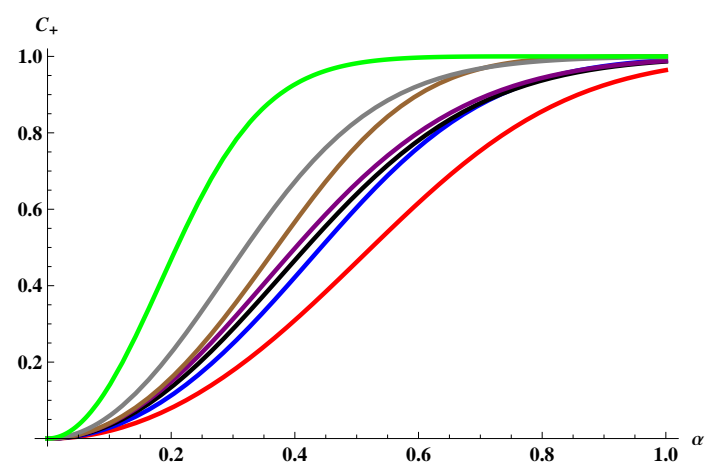

FIG. 4: (Color online) Concurrence of entanglement for the states: ECSs $\left|\Psi_{+}(\alpha, 0,0)\right\rangle$ (Red line), single-photon excited ECSs $a^{\dagger}\left|\Psi_{+}(\alpha, 0,0)\right\rangle$ (Blue line), single-mode CS-ECSs $\left|\Psi_{+}(\alpha, 1,0)\right\rangle$ (Black and Purple lines with $r=1 / \sqrt{2}, 0.6$ respectively), two-mode excited CESs $a^{\dagger} b^{\dagger}\left|\Psi_{+}(\alpha, 0,0)\right\rangle$ (Origin line), two-mode CS-CESs $\left|\Psi_{+}(\alpha, 1,1)\right\rangle$ (Gray and Green lines with $r=1 / \sqrt{2}, 0.4$ respectively).

order to describe the quality of a QT scheme, the fidelity between a unknown input state and the teleported output state is usually used as a measure. For a continuousvariable (CV) system, a teleportation scheme has been proposed according to the characteristic functions (CFs) of the quantum states concluding input, source and teleported states [33].

For a two-mode system $\rho$, the $\mathrm{CF}$ is defined as $\chi(\alpha, \beta)=\operatorname{tr}\left[D_{a}(\eta) D_{b}(\gamma) \rho\right]$, where $D_{a, b}$ are the dis- 
placement operators. Noticing the displacement operator $D_{a}(\eta)=e^{-|\eta|^{2} / 2} e^{\eta a^{\dagger}} e^{-\eta^{*} a}$ and using (3), then the $\mathrm{CF}$ of the CS-EECSs can be calculated as

$$
\begin{aligned}
\chi_{E}(\eta, \gamma) & =N_{ \pm, m, n}^{2}\left\{C F_{A}^{\alpha, \alpha}(\eta) C F_{B}^{\alpha, \alpha}(\gamma)\right. \\
& +C F_{A}^{-\alpha,-\alpha}(\eta) C F_{B}^{-\alpha,-\alpha}(\gamma) \\
& \pm e^{-4|\alpha|^{2}} C F_{A}^{\alpha,-\alpha}(\eta) C F_{B}^{\alpha,-\alpha}(\gamma) \\
& \left. \pm e^{-4|\alpha|^{2}} C F_{A}^{-\alpha, \alpha}(\eta) C F_{B}^{-\alpha, \alpha}(\gamma)\right\},
\end{aligned}
$$

where we have set (noticing $(\beta, \alpha)=( \pm \alpha, \pm \alpha)$ )

$$
\begin{aligned}
& C F_{j}^{\beta, \alpha}(\eta) \\
& =e^{-|\eta|^{2} / 2+\eta \beta^{*}-\eta^{*} \alpha} \frac{\partial^{2 m}}{\partial \tau^{m} \partial s^{m}} e^{\frac{1}{2}\left(\tau^{2}+s^{2}\right) t_{j} r_{j}} \\
& \times\left. e^{\left(\eta r_{j}+r_{j} \alpha+\beta^{*} t_{j}\right) \tau+\left(\alpha t_{j}-\eta^{*} r_{j}+r_{j} \beta^{*}\right) s+\tau s r_{j}^{2}}\right|_{s, \tau=0}, \\
& (j=A, B) .
\end{aligned}
$$

For convenience of further calculation, here we keep the differential form of $C F_{j}^{\beta, \alpha}(\eta)$ in Eq.(31).

Next, we consider the Braunstein and Kimble protocol [34] of QT for single-mode coherent-input states $|\gamma\rangle$. Note that the fidelity is independent of amplitude of the coherent state, thus for simplicity we take $\gamma=0$, then we have only to calculate the fidelity of the vacuum input state with the $\mathrm{CF} \chi_{i n}(z)=\exp \left[-|z|^{2} / 2\right]$. It is shown that, with the $\mathrm{CF} \chi_{E}(\eta, \gamma)$ for entangled channel, the CF $\chi_{\text {out }}(z)$ of the output state can be related to the CFs of input state and entangled source by formula $\chi_{\text {out }}(z)=\chi_{\text {in }}(z) \chi_{E}\left(z^{*}, z\right)$, and the fidelity of QT of CVs can be obtained as [33]

$$
\mathcal{F}=\int \frac{d^{2} z}{\pi} \chi_{\text {in }}(z) \chi_{\text {out }}(-z)
$$

Thus substituting Eqs.(30)-(31) into Eq. (32) yields

$$
\begin{aligned}
\mathcal{F}_{m, n}^{+} & =N_{+, m, n}^{2}\left[\mathcal{F}^{\alpha, \alpha}+\mathcal{F}^{-\alpha,-\alpha}\right. \\
& \left.+e^{-4|\alpha|^{2}}\left(\mathcal{F}^{\alpha,-\alpha}+\mathcal{F}^{-\alpha, \alpha}\right)\right],
\end{aligned}
$$

where we have derived

$$
\begin{aligned}
& \mathcal{F}^{\beta, \alpha} \\
& =\frac{1}{2} e^{\frac{1}{2}\left(\beta^{*}-\alpha\right)^{2}} \sum_{l=0}^{m} \sum_{f=0}^{n} \sum_{k, j=0}^{\min (n-f, m-l)} \\
& \times \frac{(m !)^{2}(n !)^{2}}{2^{m+n} l ! f ! k ! j !} \frac{(-1)^{k+j} t_{A}^{m-l} t_{B}^{n-f}}{(m-l-k) !(m-l-j) !} \\
& \times \frac{r_{A}^{m+l} r_{B}^{n+f}\left(r_{A} r_{B}\right)^{k+j}\left(\sqrt{t_{A} r_{A} t_{B} r_{B}}\right)^{-k-j}}{(n-f-k) !(n-f-j) !} \\
& \times H_{m-l-k}\left(N_{2}\right) H_{m-l-j}\left(N_{1}\right) H_{n-f-k}\left(M_{2}\right) H_{n-f-j}\left(M_{1}\right),
\end{aligned}
$$

and set

$$
\begin{aligned}
& N_{1}=\left[\beta^{*} t_{A}+\frac{1}{2} r_{A}\left(\beta^{*}+\alpha\right)\right] /\left(-i \sqrt{2 t_{A} r_{A}}\right), \\
& N_{2}=\left[\alpha t_{A}+\frac{1}{2} r_{A}\left(\beta^{*}+\alpha\right)\right] /\left(i \sqrt{2 t_{A} r_{A}}\right), \\
& M_{1}=\left[\beta^{*} t_{B}+\frac{1}{2} r_{B}\left(\beta^{*}+\alpha\right)\right] /\left(-i \sqrt{2 t_{B} r_{B}}\right), \\
& M_{2}=\left[\alpha t_{B}+\frac{1}{2} r_{B}\left(\beta^{*}+\alpha\right)\right] /\left(i \sqrt{2 t_{B} r_{B}}\right) .
\end{aligned}
$$

Eq. (33) is just the fidelity of teleporting the coherent state by using the CS-EECSs as entangled resource. In particular, when $m=n=0$, Eq. (34) reduces to $\mathcal{F}^{\beta, \alpha}=$ $\frac{1}{2} e^{\frac{1}{2}\left(\beta^{*}-\alpha\right)^{2}}$, submitting it into Eq.(33) yields

$$
\mathcal{F}_{0,0}^{+}=\frac{e^{\frac{1}{2}\left(\alpha^{*}-\alpha\right)^{2}}+e^{-4|\alpha|^{2}} e^{\frac{1}{2}\left(\alpha^{*}+\alpha\right)^{2}}}{2\left(1+e^{-4|\alpha|^{2}}\right)},
$$

which is just the fidelity by using the usual EECSs as entangled channel. It depends on the real and imagine parts of coherent amplitude $\alpha$ of the quantum channel, which is different from the case [35, 36]. If the fidelity exceeds the classical limit $1 / 2$, then the teleportation can be considered as a successful quantum protocol. From Eq.(36) it is found that under a small region of $\alpha$ $(|\alpha|<2.2), \mathcal{F}_{0,0}^{+}$may be larger than $1 / 2$ (see Fig.5) (the fidelity is always less than $1 / 2$ for odd entangled coherent state). This implies that although the odd entangled coherent state is the maximum entangled coherent state, the fidelity teleporting coherent state has not been enhanced, and that the non-maximum entangled state can be used as more effective resource for quantum teleportation [28-30].

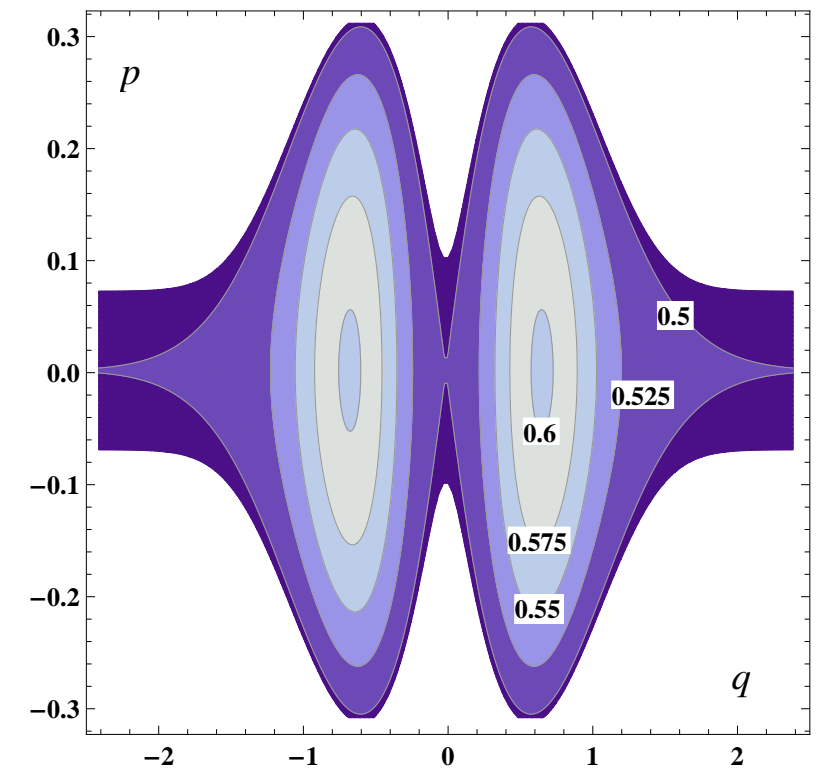

FIG. 5: (Color online) The fidelity $\mathcal{F}_{0,0}^{+}$of teleporting coherent state by using ECSs as entangled channel, where $\alpha=q+i p$.

In order to know how the CS-EECSs can improve the 
fidelity of QT, we plot the fidelity of teleporting coherent state by using CS-EECSs as the entangled resource with a symmetric case of $r_{A}=r_{B}=r(0<r<1)$ in Fig.6(a). It is shown that the maximum fidelity for $\mathcal{F}_{1,1}^{+}$ can be realized by the CS operation in the small region of coherent amplitude $\alpha$ (taking a real number) and the value of $\theta$ for optimal fidelity will be modulated by different values of small $\alpha$. Generally, the fidelity increases with the increase of the value of $\theta$. To obtain an effective QT, i.e., $\mathcal{F}_{1,1}^{+}>1 / 2, r$ must be less than $r=0.6$. For instance, from Fig.6(a) we can see that the fidelity 0.65 can be obtained well above the classical limit by the CS, say for $r=0.05$ and $\alpha=0.1$. This case is true for coherent superposition two-mode squeezed vacuum [11]. In addition, we should mention that the state $\left|\Psi_{+}(\alpha, 1,1)\right\rangle$ becomes $\left|\Psi_{+}(\alpha, 1,1)\right\rangle \sim a b\left|\Psi_{+}(\alpha, 0,0)\right\rangle \sim\left|\Psi_{+}(\alpha, 0,0)\right\rangle$ at $r=0$, thus the line of $r=0$ is just the fidelity by using the EECSs as entangled channel. From this point, we can see that for a given small value of $\alpha$ the fidelity can be optimized over the classical limit and over the fidelity at $r=0$. For the case of the odd entangled coherent state, there is no any effective fidelity found for any values of $r$ and $\alpha$.

As a comparison between the CS-EECSs and the EECSs, in Fig.6(b) we display the difference of fidelity $\left(\Delta \mathcal{F}^{+}=\mathcal{F}_{1,1}^{+}-\mathcal{F}_{0,0}^{+}\right)$as the function of $\alpha$ and $r$. It is shown that there is an over-zero region for the fidelity difference $\Delta \mathcal{F}^{+}$which means that the CS operation can be used to improve the fidelity teleporting coherent state.

In Fig. 7, we plot the fidelity as a function of $\alpha$ for several different values of $(m, n)$ with a symmetric case of $m=n$ and $r=0.195$. Form Fig.7 we can see that the fidelity $\mathcal{F}_{m, m}^{+}$decrease with the values of $m$ only when $\alpha$ exceeds a certain threshold value (Fig.7(a)). In particular, $\mathcal{F}_{2,2}^{+}$may achieve more optimal value over classical limit than $\mathcal{F}_{1,1}^{+}$in a small region of $\alpha$. On the other hand, for an asymmetric case ( $m \neq n, r=0.195)$, it is interesting to notice that the fidelity $\mathcal{F}_{m, n}^{+}$decreases due to the asymmetric operation (Fig.7(b)). These indicate that multiple CS operations may not only beat the classical bound but also even surpass single CS operations on each mode under a certain condition.

\section{CONCLUSIONS}

In this paper, we have introduced the concept of twomode coherent superposition EECSs by coherent operation $\left(r a^{\dagger}+t a\right)$ of photon subtraction and addition, and investigated the properties of entanglement according to the concurrence, the Shchukin-Vogel criteria and the average fidelity of quantum teleportation. It is shown that for the EECS, all of entanglement characteristics can be improved by local coherent operation. We made a comparison of the entanglement properties between the CS-EECSs (single-mode CS-ECSs $\left|\Psi_{+}(\alpha, 1,0)\right\rangle$ and $\left.\left|\Psi_{+}(\alpha, 1,1)\right\rangle\right)$ and the single(two-)-mode photon excited EECSs $\left(a^{\dagger}\left|\Psi_{+}(\alpha, 0,0)\right\rangle\right.$ and $\left.a^{\dagger} b^{\dagger}\left|\Psi_{+}(\alpha, 0,0)\right\rangle\right)$, which

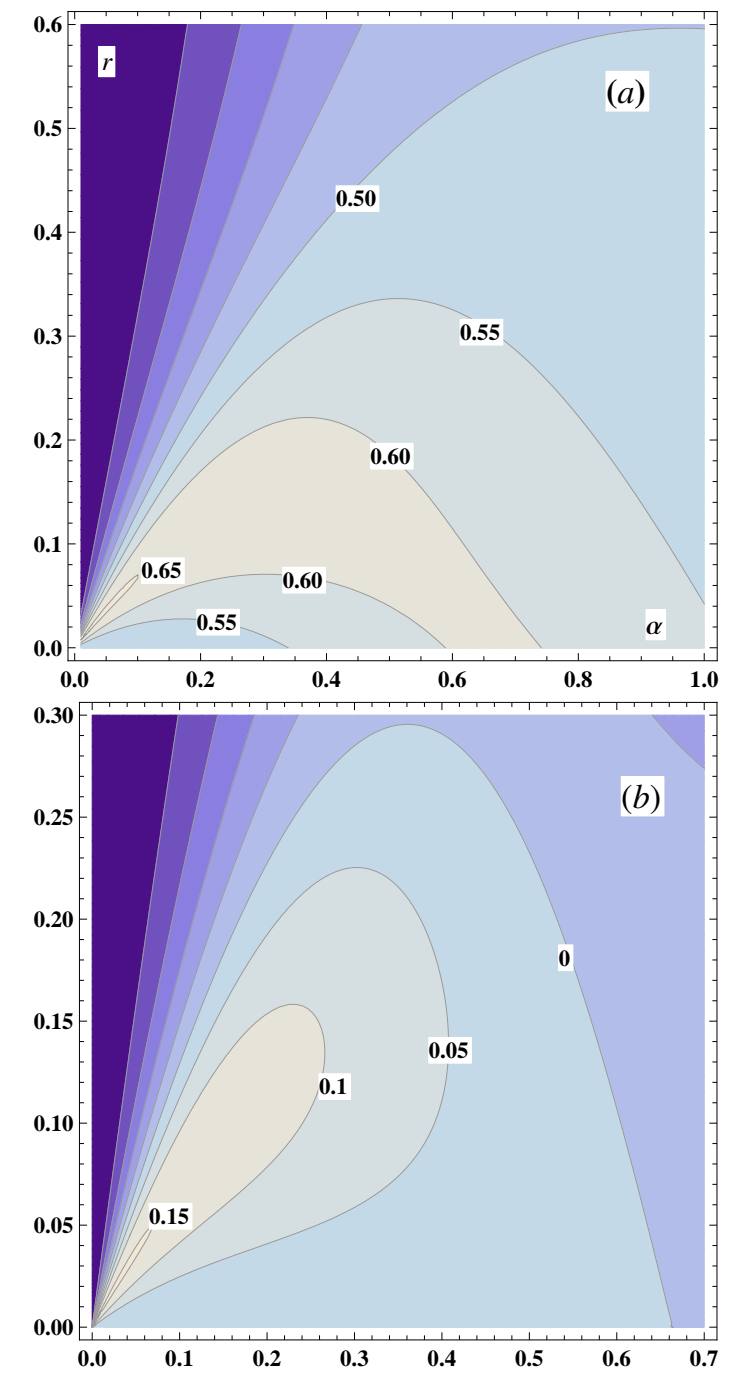

FIG. 6: (Color online) The fidelity of teleporting coherent state by using CS-EECSs as entangled channel, where $\alpha$ is a real number and $r_{A}=r_{B}=r$. (a) $F_{1,1}^{+}$as a function $\alpha$ and $r$; (b) $F_{1,1}^{+}-F_{0,0}^{+}$as a function $\alpha$ and $r$;

shows that the effects of improvement by coherent superposition operation $\left(t a+r a^{\dagger}\right)\left(t b+r b^{\dagger}\right)$ are more prominent than those by single $\left(a^{\dagger}\right)$ and two-photon $\left(a^{\dagger} b^{\dagger}\right)$ addition under a small region of amplitude, especially for an asymmetrical parameter $r$. Using the CS-EECSs with a symmetric case $\left(r_{A}=r_{B}=r\right)$ as an entangled channel, the fidelity of teleporting a coherent state is also considered, which presents that more effective quantum teleportation can be realized by $\left|\Psi_{+}(\alpha, 1,1)\right\rangle$ than $\left|\Psi_{+}(\alpha, 0,0)\right\rangle$. Although the odd entangled coherent state is a maximum entangled one, the fidelity of quantum teleportation by using it as an entangled channel is always smaller than the classical limit value $(1 / 2)$. Thus it is implied that a maximum entangled state may be not appreciate for the effective quantum teleportation.

Acknowledgments: Project supported by the National Natural Science Foundation of China (Grant 

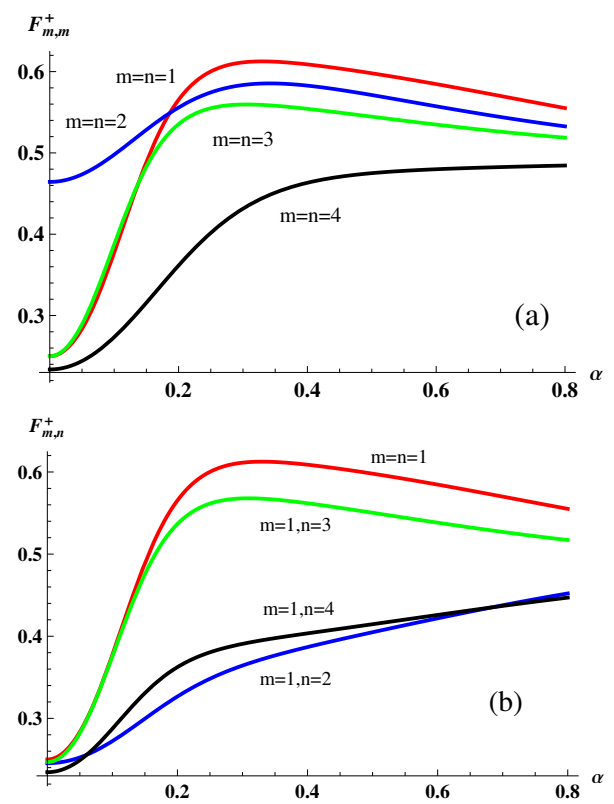

FIG. 7: (Color online) The fidelity as a function of $\alpha$ for several different values of $(m, n)$, where $\alpha$ is taken a real number and $r=0.195$.
Nos. 11264018 and 11164009), the Natural Science Foundation of Jiangxi Province of China (Grant No. 20132BAB212006), and the Research Foundation of the Education Department of Jiangxi Province of China (no GJJ14274) as well as Degree and postgraduate education teaching reform project of jiangxi province(No. JXYJG-2013-027).
[1] D.Bouwmeester, et al.The Physics of Quantum Information. Springer, Berlin (2000).

[2] M. S. Kim, "Recent developments in photon-level operations on travelling light fields," J. Phys. B 41, 133001133018 (2008).

[3] Alexei Ourjoumtsev, Aurelien Dantan, Rosa Tualle-Brouri, and Philippe Grangier, "Increasing Entanglement between Gaussian States by Coherent Photon Subtraction," Phys. Rev. Lett. 98, 030502 (2007).

[4] A. Ourjoumtsev, R. Tualle-Brouri, P. Grangier, "Quantum Homodyne Tomography of a Two-Photon Fock State," Phys. Rev. Lett. 96, 213601 (2006).

[5] Y. Yang, L. F. Li, "Nonclassicality of photon-subtracted and photon-added-then-subtracted Gaussian states," J. Opt. Soc. Am. B, 26, 830-835 (2009).

[6] L. Y. Hu, Z. M. Zhang, "Statistical properties of coherent photon-added two-mode squeezed vacuum and its inseparability," J. Opt. Soc. Am. B 30, 286518-529 (2013).

[7] H. L. Zhang, Y. Q. Hu, F. Jia, L. Y. Hu, "Entanglement of Photon-Subtracted Two-Mode Squeezed Thermal State and Its Decoherence in Thermal Environments," Int. J. Theor. Phys. 53, 2091-2107 (2014).

[8] Su-Yong Lee, Jiyong Park, Se-Wan Ji, C. H.Raymond Ooi, and Hai-Woong Lee, "Nonclassicality generated by photon annihilation-then-creation and creation-then-annihilation operations," J. Opt. Soc. Am. B 26, 1532-1537 (2009).

[9] Z. Wang, H. C. Yuan, and H. Y. Fan, "Photon-subtracted squeezed coherent state: nonclassicality and decoherence in thermal environment," J. Opt. Soc. Am. B 1964-1972 (2011).

[10] S. Y. Lee and H. Nha, "Quantum state engineering by a coherent superposition of photon subtraction and addition," Phys. Rev. A 82, 053812 (2010).
[11] S. Y. Lee, S. W. Ji, H. J. Kim and H. Nha, "Enhancing quantum entanglement for continuous variables by a coherent superposition of photon subtraction and addition," Phys. Rev. A 84, 012302 (2011).

[12] S. Y. Lee, S. W. Ji and C. W. Lee, "Increasing and decreasing entanglement characteristics for continuous variables by local photon subtraction," Phys. Rev. A 87, 052321 (2013).

[13] Barry C Sanders, "Review of entangled coherent states," J. Phys. A: Math. Theor. 45, 244002 (2012).

[14] Dong-Lin Zhou and Le-Man Kuang, "Two-mode excited entangled coherent states and their entanglement properties," Chin. Phys. B 18, 1328 (2009).

[15] J. S. Zhang and J. B. Xu, "Entanglement and nonlocality of photon-added entangled coherent states and quantum probabilistic teleportation," Phys. Scr. 79, 025008 (2009).

[16] H C Yuan, L. Y. Hu, "Comment on "Single-mode excited entangled coherent states," J. Phys. A: Math. Theor. 43, 018001 (2010).

[17] L. Y. Hu, F. Jia and Z. M. Zhang, "Entanglement and nonclassicality of photon-added two-mode squeezed thermal state," J. Opt. Soc. Am. B 29, 1456 (2012).

[18] E. Shchukin and W. Vogel, "Inseparability criteria for continuous bipartite quantum states," Phys. Rev. Lett. 95, 230502 (2005).

[19] X. Y. Chen, "The entanglement properties of non-Gaussian states prepared by photon subtraction from two-mode squeezed thermal states," Phys. Lett. A 372, 2976-2979 (2008).

[20] A. Mann, B.C. Sanders, W.J. Munro, "Bell's inequality for an entanglement of nonorthogonal states," Phys. Rev. A 51, 989 (1995).

[21] S. Hill, W.K. Wootters, "Entanglement of a Pair of Quan- 
tum Bits," Phys. Rev. Lett. 78, 5022 (1997).

[22] W. K. Wootters, "Entanglement of Formation of an Arbitrary State of Two Qubits," Phys. Rev. Lett. 80, 2245 (1998).

[23] X. G. Wang, "Quantum teleportation of entangled coherent states," Phys. Rev. A 64, 022302 (2001).

[24] X. G. Wang, "Bipartite maximal entangled nonorthogonal states," J. Phys. A: Math. Gen. 35, 165-173 (2002).

[25] C. Invernizzi, S. Olivares, M. G. A. Paris, and K. Banaszek, "Effect of noise and enhancement of nonlocality in on/off photo detection," Phys. Rev. A 72, 042105042116 (2005).

[26] A. Kitagawa, M. Takeoka, M. Sasaki, and A. Chefles, "Entanglement evaluation of non-Gaussian states generated by photon subtraction from squeezed states," Phys. Rev. A 73, 042310-042321 (2006).

[27] T. Opatrny, G. Kurizki, and D.-G. Welsch, "Improvement on teleportation of continuous variables by photon subtraction via conditional measurement," Phys. Rev. A 61, 032302 (2000).

[28] L. Y. Hu, X. X. Xu, and H. Y. Fan, "Statistical properties of photon-subtracted two-mode squeezed vacuum and its decoherence in thermal environment," J. Opt. Soc. Am. B 27, 286-299 (2010).

[29] S. Olivares, M. G. A. Paris, and R. Bonifacio, "Teleportation improvement by inconclusive photon subtraction," Phys. Rev. A 67, 032314-032318 (2003).
[30] P. T. Cochrane, T. C. Ralph, and G. J. Milburn, “Teleportation improvement by condition measurements on the two-mode squeezed vacuum," Phys. Rev. A 65, 062306062311 (2002).

[31] S. D. Bartlett and B. C. Sanders, "Universal continuous variable quantum computation: requirement of optical nonlinearity for photon counting," Phys. Rev. A 65, 042304-042308 (2002).

[32] M. Sasaki and S. Suzuki, "Multimode theory of measurement induced non-Gaussian operation on wideband squeezed light: analytical formula," Phys. Rev. A 73, 043807-043824 (2006).

[33] P. Marian and T. A. Marian, "Continuous-variable teleportation in the characteristic-function description," Phys. Rev. A 74, 042306 (2006).

[34] S. L. Braunstein and H. J. Kimble, "Teleportation of continuous quantum variables," Phys. Rev. Lett. 80, 869-872 (1998).

[35] H. Jeong, M. S. Kim and J. Lee, "Quantum-information processing for a coherent superposition state via a mixed entangled coherent channel," Phys. Rev. A, 64, 052308 (2001).

[36] J. Lee, M. S. Kim and H. Jeong, "Transfer of nonclassical features in quantum teleportation via a mixed quantum channel," Phys. Rev. A, 62, 032305 (2000). 